\title{
Substrate elasticity modulates TGF beta stimulated re-differentiation of expanded human articular chondrocytes
}

\author{
Daniel Vonwil • Andreas Trüssel • Olivia Haupt • \\ Samy Gobaa • Andrea Barbero • V. Prasad. Shastri • \\ Ivan Martin
}

(C) Controlled Release Society 2012

\begin{abstract}
Substrate elasticity has emerged as important biomaterial design parameter. In particular, it has been reported that on soft substrates $(\sim 4 \mathrm{kPa})$ freshly isolated porcine chondrocytes better maintain their phenotype than on stiffer ones $(>20 \mathrm{kPa})$. Thus, we investigated whether this also applies to re-differentiating, expanded/de-differentiated (EDD) human articular chondrocytes (HAC). EDD HAC were seeded onto Type I collagen functionalized poly acrylamide (PA) films with a Young's modulus of $0.26 \pm 0.08 \mathrm{kPa}$ (soft), $21.32 \pm 0.79 \mathrm{kPa}$ (intermediately stiff) and $74.88 \pm$ $5.13 \mathrm{kPa}$ (stiff), or type I collagen-coated plastic dishes (TCPS w/CI). Cells were cultured for 7 to 14 days in chondrogenic medium supplemented with transforming growth factor beta-1 (TGF- $\beta 1$ ) and assessed for attachment, initial adhesion strength, proliferation, morphology as well as for expression of type I and II collagen at mRNA and type II collagen on protein level. Attachment and adhesion strength was similar on the different PA substrates and
\end{abstract}

D. Vonwil • O. Haupt • A. Barbero · I. Martin $(\bowtie)$

Department of Surgery and of Biomedicine,

University Hospital Basel,

Hebelstrasse 20,

4031 Basel, Switzerland

e-mail: IMartin@uhbs.ch

D. Vonwil

e-mail:vonwilda@gmail.com

O. Haupt

e-mail: olivia.haupt@spirig.ch

A. Barbero

e-mail: abarbero@uhbs.ch

\section{A. Trüssel}

ETH Zürich, Institut für Biomechanik,

Wolfgang-Pauli-Str. 10,

8093 Zürich, Switzerland

e-mail: atruessel@ethz.ch proliferation remained marginal $(<1$ doubling/week). On intermediately stiff to infinitely stiff substrates EDD HAC assumed a spindle shaped, fibroblastic morphology, whereas on the soft substrate they remained more spherical, as assessed by shape factor analysis, and had a reduced spreading area (up to 3.2-fold). F-actin organization on the soft substrate was restricted cortically, while on the stiffer substrates F-actin assembled into stress fibers. While type II collagen mRNA expression on the soft substrate was (similar to that in aggregate culture and) 18 -fold higher than on TCPS w/CI, it was not detectable on protein level. On all substrates, in the absence of TGF- $\beta 1$ type II collagen mRNA remained at levels expressed by EDD HAC. In summary, substrate elasticity modulated the TGF- $\beta 1$ stimulated re-differentiation of EDD HAC. Mechanical compliance is thus an important parameter to be coupled with the delivery of appropriate morphogens in designing biomaterials for cartilage engineering and repair.

\section{S. Gobaa}

Laboratory of Stem Cell Bioengineering,

Station 19 ,

1015 Lausanne, Switzerland

e-mail: samy.gobaa@epfl.ch 
Keywords Biomaterial interface $\cdot$ Regenerative medicine Cartilage repair - Cartilage differentiation

\section{Introduction}

Current visions in the treatment of cartilage defects aim at operating a shift from a fibrocartilaginous to a more hyaline like repair tissue by combining repair supporting biomaterials with autologous cells as for example mesenchymal stem cells or articular chondrocytes [1]. In this paradigm, the role of a chondro-supportive biomaterial within a cartilage defect is seen to support chondrogenic differentiation and to protect/modulate the newly formed tissue [2]. Apart from controlling ligand identity, density, and presentation, which determine biomaterial/cell interaction [3], substrate elasticity has emerged as an important, instructive cue and has been shown to influence the behavior of mesenchymal stem cells [4] and that of more specified members of mesenchymal origin including fibroblasts [5,6], myoblasts [7-9], and osteoblasts [10]. More recently, Schuh et al. have reported that the phenotype of porcine articular chondrocytes is better maintained on soft ( $4 \mathrm{kPa})$ as compared to on stiff substrates $(100 \mathrm{kPa})$ [11]. In that study, the reduced maintenance of chondrogenic phenotype on the stiffer substrates was accompanied by increased proliferation, which is known to lead to rapid de-differentiation in chondrocytes [12]. General hallmarks of chondrocyte de-differentiation, which is generally associated with monolayer expansion, are a reduced production of type II collagen and the large proteoglycan aggrecan $[13,14]$ as well as a switch to the synthesis of type I collagen and fibronectin [15]. The proliferation observed by Schuh et al. [11] likely has been induced by serum (10\%) in the culture medium and could have masked the effect of substrate elasticity on chondrocyte phenotype. This prompts for the use of a culture medium that does not stimulate cell proliferation.

Proliferation associated de-differentiation can further be accelerated by the use of specific growth factors during monolayer expansion of HAC and was proposed to induce a differentiation plasticity in HAC which is similar to that of mesenchymal progenitor stem cells [16]. Yet, culturing aggregates (suspension or pellet culture) of expanded/dedifferentiated (EDD) HAC in a medium containing the strong chondrogenic stimulus transforming growth factor beta-1 (TGF- $\beta 1$ ), allows for re-induction of chondrogenic differentiation and manifests itself by a reversion of the hallmarks mentioned above $[17,18]$.

Similar to Schuh et al. [11], we wanted to assess whether the chondrogenic phenotype is better supported on soft as compared to on stiffer substrates. However, instead of maintaining the chondrogenic phenotype of freshly isolated chondrocytes, we focused on the re-differentiation of EDD
HAC in the presence of the soluble chondrogenic differentiation signal TGF- $\beta 1$. Furthermore, Engler et al. have reported that even in the absence of exogenous soluble stimuli, matrix elasticity stimulates mesenchymal stem cells to up-regulate genes specific for different lineages [4]. Based on this finding and on the progenitor-like differentiation plasticity mentioned for EDD HAC [16], we also wanted to investigate whether in absence of TGF- $\beta 1$ a soft substrate per se would allow to up-regulate the key chondrogenic gene type II collagen.

To avoid proliferation associated de-differentiation, which could have masked the investigation of matrix elasticity in the previously mentioned study [11], a defined serum-free medium (SFM) was chosen. SFM is considered as a standard in chondrogenic culture and does not appear to stimulate mitotic activity EDD HAC [16, 19].

The adhesion, proliferation, morphology, gene (types I and II collagen) and protein expression (type II collagen) of re-differentiating EDD HAC was assessed on collagen type I-coated polyacrylamide (PA) substrates with different stiffness. This substrate model has been chosen since it can easily be adjusted in its stiffness and has been widely used in the field of mechanobiology $[6,7,11,20-22]$. Although PA itself does not promote cell adhesion, it can be functionalized by covalently grafting proteins to its surface [23]. This offers the advantage to restrict cell/substrate interaction to a selected type of ligand. For the present study, type I collagen (CI) was chosen as it contains the cell adhesion peptide sequence Arg-Gly-Asp (RGD) [24], has been used previously to study the influence of matrix elasticity on the lineage specification of mesenchymal stem cells [4] as well as on the phenotype of articular chondrocytes [11] and plays an important role during limb bud chondrogenesis [25].

\section{Methods}

\section{Substrate preparation}

PA gels of three different, contrasting compositions, were cast onto 3-(trimethoxysilyl)-propyl methacrylate (Sigma-Aldrich, Buchs, $\mathrm{CH}$ ) activated glass slides, and functionalized with type I collagen following a protocol adapted from Beningo et al. $2002[22,26]$. The polymerization was allowed to complete for $>4 \mathrm{~h}$ and the gel thickness set to a thickness ranging from 100 to $170 \mu \mathrm{m}$ using coverglass spacers (No. 1, Medite, $\mathrm{CH}$ ). Subsequently, the glass immobilized PA gels were washed with autoclaved MilliQ water followed by 50 mM HEPES pH 8.5 (Sigma-Aldrich, Buchs, CH). The PA gels were covered with $1 \mathrm{mM}$ sulfo-SANPAH (heterobifunctional protein crosslinker, ProteoChem, USA) in $50 \mathrm{mM}$ HEPES pH 8.5 and UV activated $(350 \mathrm{~nm} ; 8 \mathrm{~min}$; distance, 
$6 \mathrm{~cm})$. This step was repeated once before thoroughly washing the substrates with PBS (Gibco, UK). Immediately after, the activated PA gels were exposed to $0.2 \mathrm{mg} / \mathrm{ml}$ type I collagen (CI, rat tail, BD, UK) at $4{ }^{\circ} \mathrm{C}$ overnight. Collagen I-coated tissue culture-treated plastic (TCPS w/CI) was prepared as described for activated PA gels. All substrates were UVlight-sterilized for $30 \mathrm{~min}$ in a sterile work bench, washed with PBS and stored at $4{ }^{\circ} \mathrm{C}$ until further use.

Substrate characterization by rotational rheometry

The polymerization time course (20 min, see Fig. 1) of three different mixtures consisting of acrylamide (AA, monomer) and $N, N^{\prime}$-methylenbisacrylamide (BIS, crosslinker) was followed in situ in a rotational rheometer (Physica MCR 501, Anton Paar, Graz, Austria), fitted with a 50-mm diameter parallel plate (PP50). Shear storage modulus $\left(G^{\prime}\right)$ measurements were conducted at room temperature $\left(25^{\circ} \mathrm{C}\right)$, an oscillation frequency of $1 \mathrm{~Hz}$ and an amplitude of $1 \%$. The volume for polymerization was $2 \mathrm{ml}$ and initiated with $30 \mu \mathrm{l}$ of $10 \%$ ammonium persulfate and $30 \mu$ of $10 \%$ tetramethylethylenediamine. The concentrations $(\% w / v)$ for AA_BIS were: 5\%_0.003\%, 10\%_0.2\%, 20\%_0.3\% as described above and the duplicates prepared independently. To prevent drying, polymerization was performed in a humidified atmosphere.

PA slabs of $4 \mathrm{~mm}$ thickness were polymerized for $>4 \mathrm{~h}$ and placed in PBS at room temperature $>24 \mathrm{~h}$. The elasticity of swollen (equilibrium) PA gels was determined with rotational rheometry by measuring the dynamic shear modulus $(G)$, which is the sum of the two components, $G^{\prime}$ and the shear loss modulus ( $G^{\prime \prime}$, see Table 2$)$. Using the equation:

$E=2 G^{\prime}(1+v)$

the dynamic shear modulus was converted into the more commonly used Young's modulus (see Table 2). For the

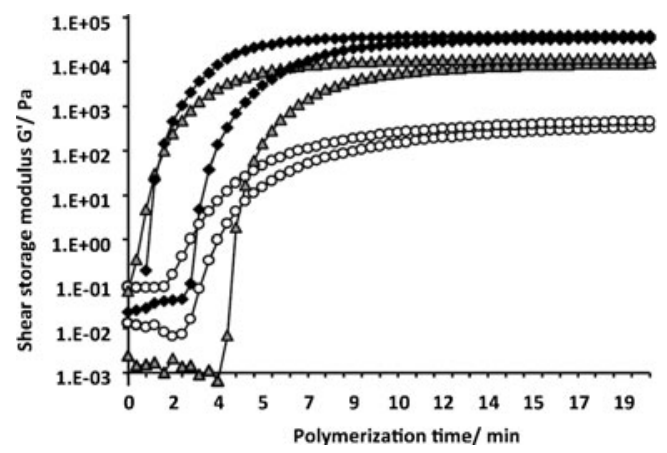

Fig. 1 Time course of shear storage modulus $\left(G^{\prime}, \mathrm{Pa}\right)$ in polymerizing acrylamide (PA) mixtures. The polymerization time course $(20 \mathrm{~min})$ of three different mixtures consisting of acrylamide (AA, monomer) and $N, N^{\prime}$-methylenbisacrylamide (BIS, crosslinker) was followed in situ in a rotational rheometer. The concentrations $(w / v)$ for AA_BIS were: $5 \% 0.003 \%$ (open circles), $10 \%$ \% $0.2 \%$ (gray triangles), $20 \% 0.3 \%$ (black diamonds). Duplicates were prepared independently
Poisson ratio $(\nu)$ a value of 0.464 was assumed as it has been reported for PA by others [27].

\section{Cell culture}

Full thickness human articular cartilage samples were collected within $24 \mathrm{~h}$ post mortem from the femoral lateral condyle of four donors (mean age, 60 years; age range, 43-77 years), with no history of joint disease, after obtained informed consent, following protocol approval by the local ethical committee (No. 78/07). HAC were isolated upon 22-hour incubation at $37^{\circ} \mathrm{C}$ in $0.15 \%$ type II collagenase and re-suspended with Dulbecco's modified Eagle's medium (DMEM) containing $4.5 \mathrm{mg} / \mathrm{ml} \mathrm{D}$-glucose, $0.1 \mathrm{mM}$ non-essential amino acids, $1 \mathrm{mM}$ sodium pyruvate, $10 \mathrm{mM}$ HEPES buffer, $100 \mathrm{U} / \mathrm{ml}$ penicillin, $100 \mu \mathrm{g} / \mathrm{ml}$ streptomycin, and $0.29 \mathrm{mg} / \mathrm{mlL}$-glutamine, supplemented with $10 \%$ FBS (all from Gibco, UK) (complete medium, CM). HAC were expanded in CM supplemented with $1 \mathrm{ng} / \mathrm{ml}$ TGF- $\beta 1,5 \mathrm{ng} / \mathrm{ml}$ FGF-2, $10 \mathrm{ng} / \mathrm{ml}$ PDGF-BB (expansion medium, all from R\&D, UK) in a humified $37{ }^{\circ} \mathrm{C} / 5 \% \mathrm{CO}_{2}$ incubator. These specific growth factors have previously been shown to enhance HAC proliferation and post-expansion re-differentiation capacity [18]. HAC were detached at confluency (passage 1) by a treatment with $0.3 \%$ type II collagenase, followed by $0.05 \%$ trypsin/ $0.52 \mathrm{mM}$ EDTA (Gibco, UK) and re-plated at a density of $5 \times$ $10^{3}$ cells $/ \mathrm{cm}^{2}$ for the second expansion step. After passage 3 to 4, expanded, de-differentiated (EDD) HAC were taken into chondrogenic re-differentiation culture.

The chondrogenic re-differentiation of EDD HAC was stimulated using a chemically defined, serum-free medium (SFM), which consists of DMEM containing $4.5 \mathrm{mg} / \mathrm{ml} \mathrm{D-}$ glucose, $0.1 \mathrm{mM}$ non-essential amino acids, $1 \mathrm{mM}$ sodium pyruvate, $10 \mathrm{mM}$ HEPES buffer, $100 \mathrm{U} / \mathrm{ml}$ penicillin, $100 \mu \mathrm{g} / \mathrm{ml}$ streptomycin, and $0.29 \mathrm{mg} / \mathrm{mlL}$-glutamine (all Gibco, UK) supplemented with $\operatorname{ITS}^{+1}(10 \mu \mathrm{g} / \mathrm{ml}$ insulin, $5.5 \mathrm{mg} / \mathrm{ml}$ transferrin, $5 \mathrm{ng} / \mathrm{ml}$ selenium, $0.5 \mathrm{mg} / \mathrm{ml}$ bovine serum albumin, $4.7 \mathrm{mg} / \mathrm{ml}$ linoleic acid), $1.25 \mathrm{mg} / \mathrm{ml}$ human serum albumin, $0.1 \mathrm{mM}$ ascorbic acid 2-phosphate and $10^{-7} \mathrm{M}$ dexamethasone (all Sigma Chemical, USA) and $10 \mathrm{ng} / \mathrm{ml}$ TGF- $\beta 1$ (R\&D, UK; chondrogenic medium) [16, 19]. To investigate the influence of substrate stiffness on EDD HAC re-differentiation, EDD HAC were cultured for 7 days on type I collagen-coated PA gels of different stiffness at density of $2 \times 10^{4}$ cells $/ \mathrm{cm}^{2}$. Aggregate cultures were prepared by culturing EDD HAC at the same density on TCPS without collagen I coating (TCPS w/o CI). Due to the absence of serum proteins in SFM, HAC display low adhesion to TCPS w/o CI and preferentially stick to each other rather than to the substrate. After 1 day of re-differentiation culture on TCPS w/o CI, nearly all HAC have detached from the substrates and float in suspension as cellular aggregates similarly as described previously [17]. 


\section{Cell attachment and proliferation}

The number of adherent HAC $\left(N_{0}\right)$ was determined from phase contrast light microscopy images and expressed as percentage of the number of theoretically seeded cells. After 7 days of re-differentiation culture, the number of adherent HAC $\left(N_{1}\right)$ was counted again (as described above) to assess proliferation as expressed by the total number of doublings: $\log _{2}\left(N_{1} / N_{0}\right)$. The number of cells was counted in two representative images $\left(\times 20\right.$ objective; $150 \mathrm{\mu m}^{2} /$ field $)$ of each substrate for three different donors.

Initial adhesion strength

For each stiffness, four substrates were prepared. HAC were seeded onto the four substrates with a density of $2 \times 10^{4}$ cells $/ \mathrm{cm}^{2}$. At seven different time points (5-300 $\mathrm{min}$ ) two of the substrates were fixed with $4 \%(w / w)$ formaldehyde in phosphate buffer ( $\mathrm{pH}$ 7.4) on ice (static controls). The other two were placed upside down on TeflonR rings in six well plates containing PBS, centrifuged at 3,044 rpm (Heraeus Multifuge 3SR+, Thermo Science, Waltham USA) and also fixed as described for the static controls.

According to Channavajjala et al. [28] and Maheshwari et al. [29], the pulling force $F$ (Eq. 2) acting on the cell is:

$$
\begin{aligned}
F & =V_{\mathrm{c}} \times\left(\rho_{\mathrm{c}}-\rho_{\mathrm{m}}\right) \times r \times \omega^{2} \\
& =V_{\mathrm{c}} \times\left(\rho_{\mathrm{c}}-\rho_{\mathrm{m}}\right) \times \mathrm{RCF}
\end{aligned}
$$

where $V_{\mathrm{c}}$ is the volume of the cell, $\rho_{\mathrm{c}}$ is the mean density of the intracellular space, $\rho_{\mathrm{m}}$ the density of the medium, RCF is the relative centrifugation force, $\omega$ the angular speed, and $r$ the rotor radius. We assumed, that the volume of the cell $V_{\mathrm{c}}$ corresponds to a sphere with a diameter of $13 \mu \mathrm{m}$ (according to the cell counter and microscopy), the density of the cell $\rho_{\mathrm{c}}$ was $1.075 \mathrm{~g} / \mathrm{ml}$ [30] and that of the medium $\rho_{\mathrm{m}}$ was $1.00 \mathrm{~g} / \mathrm{ml}$. An angular speed of 3,044 rpm was applied resulting in an RCF of 1,627 $\mathrm{g}$. Under these assumptions the force $F$ acting on a cell was $1.38 \mathrm{nN}$.

The number of adherent HAC was determined semi automatically by counting DAPI-stained nuclei. Fluorescence images were taken on each slide at four random positions. ImageJ [31] was used to count the nuclei. The adherent cell fraction was determined by normalizing the cell number on the centrifuged substrates to the static controls and plotted over time. In general, these plots showed a monotone increasing function with a plateau at later time points. Reyes et al. [30] showed that the adherent cell fraction over surface ligand density showed a sigmoidal characteristic. We have adapted this formula to calculate the adherent cell fraction (acf, Eq. 3) as function of adhesion time. $\operatorname{acf}(t)=\operatorname{acf}_{\max } \times\left(1+\exp \left(-\left(t-t_{50}\right) / b\right)\right)^{-1}$

Where $\operatorname{acf}(t)$ is the adherent cell fraction at time point $t, \operatorname{acf}_{\max }$ is the adherent cell fraction at an infinite time point, $b$ is the maximal slope of the curve and $t_{50}$ is the time point, at which $50 \%$ of the cells were adherent after centrifugation. This $t_{50}$ served as quantitative value for adhesion and was lower; the faster cells were able to adhere.

\section{Cell morphology}

HAC morphology and the degree of spreading were determined after $24 \mathrm{~h}$ of re-differentiation culture. The cell shape factor $\phi$ (Eq. 4) [32] was calculated according to:

$\phi=\frac{4 \pi \mathrm{A}}{\mathrm{p}^{2}}$

Rounded cells with high area-to perimeter ratio assume shape factor $\phi$ values close to 1 , whereas starfish-shaped cells have a shape factor $\phi$ near 0 . The area $\mathrm{A}$ and the perimeter $\mathrm{p}$ to calculate the shape factor $\phi$ were derived from phase contrast images using Image $\mathrm{J}$ version 1.37 [31]. All cells in two representative images $(\times 20$ objective; $150 \mathrm{\mu m}^{2} /$ field) of each substrate type were analyzed for three different donors.

Fluorescence labeling, acquisition and analysis

After fixation in $4 \%(w / w)$ formaldehyde in phosphate buffer pH 7.4 (University Hospital Pharmacy Basel, Switzerland) at $4{ }^{\circ} \mathrm{C}$ overnight, specimens were rinsed with PBS three times prior to a $10-\mathrm{min}$ permeabilization on ice with a solution containing $0.02 \%(w / w)$ Triton X100 (SigmaAldrich, Switzerland). Immediately after aspiration of the permeabilization solution, the specimens were blocked for $1 \mathrm{~h}$ at room temperature in PBS containing $30 \mathrm{mg} / \mathrm{ml} \mathrm{BSA}$ (Sigma-Aldrich, Switzerland). Then, the specimens were rinsed with labeling buffer (LB) containing $15 \mathrm{mg} / \mathrm{ml} \mathrm{BSA}$ in PBS and incubated with the primary antibody for $1 \mathrm{~h}$ at room temperature. Subsequently, the specimens were rinsed with LB four times for $5 \mathrm{~min}$ each and incubated with the secondary antibody for $1 \mathrm{~h}$ at room temperature. Finally, the specimens were washed again with LB four times for $5 \mathrm{~min}$ each, rinsed with autoclaved milliQ water, mounted with aqueous mounting media (AbD SeroTec, Oxford, UK) and sealed with Klarlack (Lady Manhattan Cosmetics, Germany). All antibodies/labeling agents were diluted in LB. Vinculin was labeled with a primary monoclonal mouse antibody (Sigma-Aldrich, Switzerland; clone: hVIN-1; dilution: 1:400) followed by a Cy3 conjugated $\operatorname{IgG}$ goat antimouse secondary antibody (Acris Antibodies, Herford Germany; dilution, 1:800). F-actin was detected with Alexa488 
conjugated phalloidin (Invitrogen, Oregon USA; dilution, 1:400) and nuclei stained with DAPI (Invitrogen, Oregon USA; 4',6-diamidino-2-phenylindole; dilution, 1:48,000). Type II collagen was labeled with a primary monoclonal mouse antibody (MP Biomedicals, Germany, clone II-4C11; dilution, 1:1000) followed by $\mathrm{Cy} 3$ conjugated anti-mouse antibody as described for vinculin.

Fluorescence images were acquired on a Zeiss LSM 710 TYPE (Zeiss, Wetzlar, Germany) confocal laser-scanning microscope under constant conditions using either a $\times 40$ or a $\times 63$ immersion objective. Eight bit z-stack images were recorded in three different, regions of each substrate.

Gene expression analysis

After 7 days of re-differentiation culture, cells were harvested (as described above; using collagenase and trypsin) and the RNA extracted from the obtained cell pellets with $250 \mu \mathrm{l}$ Trizol (Life Technologies, Basel, $\mathrm{CH}$ ) according to the manufacturer's instructions. Extracted RNA was treated with DNAse following the instructions of the RNeasy Kit (Ambion, Austin TX). cDNA was generated from total RNA using reverse-transcriptase Stratascript (Stratagene) in the presence of dNTP and DTT. Real-time PCR reactions were performed and monitored using the ABI prism 7700 Sequence Detection System and the Sequence Detector V program (Perkin-Elmer Applied Biosystems). cDNA samples were analyzed for type I and II collagen and for the housekeeping gene (18S ribosomal RNA), using previously described sequences of primers and probes (see Table 1) [16]. Each cDNA sample was assessed in duplicate and the collagen mRNA expression levels were normalized to the corresponding $18 \mathrm{~S}$ rRNA levels.

\section{Collagen western blot}

HAC were cultured on the corresponding substrates at a density of $3 \times 10^{4}$ cells $/ \mathrm{cm}^{2}$ in chondrogenic medium for 14 days, harvested using collagenase/trypsin (as described above) and lysed with Laemmli buffer. The native cartilage (positive control) and HAC cultured in aggregates were heated in Laemmli buffer to complete lysis. The protein content of the samples was determined with a BCA Protein Assay Kit (Thermo Fisher Scientific, Rockford, United States). Equal amounts of protein were separated on $10 \%$ SDS gels. After the electrophoresis the gels were blotted on PVDF membranes for $3 \mathrm{~h}$ at $4{ }^{\circ} \mathrm{C}$ and $0.33 \mathrm{~A}$. Membranes were stained with Ponceau S Red to check the transfer of the proteins and destained thereafter. The membranes were blocked for $1 \mathrm{~h}$ with $0.2 \%$ Blocking Reagenz CA (AppliChem, Darmstadt, Germany) in PBS with $0.1 \%$ Tween 20 (blocking solution). Subsequently, the membranes were incubated with a monoclonal mouse antibody against human type II collagen (MP Biomedicals, Germany, Clone: II4 C11) 1:2,000 diluted in blocking solution. The membranes were washed three times for 10 min with PBS $0.1 \%$ Tween (PBST) and incubated for $1 \mathrm{~h}$ with the secondary antibody (goat anti-mouse IgG HRP conjugated, Thermo Fisher scientific, Rockford, United States) diluted 1:5,000 in PBST. Again the membranes were washed three times for $10 \mathrm{~min}$ in PBST. The blots were developed using a bioluminescence system (SuperSignal West Pico, Thermo Fisher Scientific, Rockford, United States) according to manufacturer's instructions. Bands were detected with the Fusion Fx7 imager (Peqlab, Erlangen, Germany).

\section{Statistical analysis}

Statistical evaluation was performed using the free online statistics program BrightStat [33]. All values are presented as mean \pm standard error (SE). Differences were analyzed using complying statistical tests as indicated at each result. In general, $n$ was low and prompted for distribution free analysis. Mann-Whitney (comparison between two groups; effect $+/-$ TGF- $\beta 1$ ) and Kruskal-Wallis (comparison between more than two groups; effects on different substrates) were the non-parametric tests of choice. For cell morphology however, $n$ was large enough and the data followed a normal distribution, which allowed for parametrical analysis by one-way ANOVA. For all tests, the level of significance was set to $p<0.05$.

Table 1 Description of the designed primers and probes for real-time RT-PCR

\begin{tabular}{|c|c|c|c|c|}
\hline Gene & Forward and reverse primes $\left(5^{\prime} \rightarrow 3^{\prime}\right)$ & Primer concentration (nM) & Probe $\left(5^{\prime} \rightarrow 3^{\prime}\right)$ & Probe concentration $(\mathrm{nM})$ \\
\hline $18 \mathrm{~S}$ rRNA & $\begin{array}{l}\text { CGGCTACCACATCCAAGGAA } \\
\text { GCTGGAATTACCGCGGCT }\end{array}$ & 26 & $\begin{array}{l}\text { TGCTGGCACCA } \\
\text { GACTTGCCCTC }\end{array}$ & 50 \\
\hline Type I collagen & $\begin{array}{l}\text { TTTTGTATTCAATCACTGTCTTGCC } \\
\text { CAGCCGCTTCACCTACAGC }\end{array}$ & 300 & $\begin{array}{l}\text { CCGGTGTGACT } \\
\text { CGTGCAGCCATC }\end{array}$ & 100 \\
\hline Type II collagen & $\begin{array}{l}\text { GGCAATAGCAGGTTCACGTACA } \\
\text { CGATAACAGTCTTGCCCCACTT }\end{array}$ & 900 & $\begin{array}{l}\text { CCGGTATGTTTC } \\
\text { GTGCAGCCATCCT }\end{array}$ & 200 \\
\hline
\end{tabular}


Table 2 Rheology of polyacrylamide (PA) gels

\begin{tabular}{|c|c|c|c|c|c|c|}
\hline \multirow{2}{*}{$\begin{array}{l}\text { Composition } \\
\text { AA/BIS (\%) }\end{array}$} & \multicolumn{3}{|c|}{ In situ polymerized } & \multicolumn{3}{|c|}{ Swelling equilibrated } \\
\hline & $E(\mathrm{kPa})$ & $G^{\prime}(\mathrm{kPa})$ & $G^{\prime \prime}(\mathrm{Pa})$ & $E(\mathrm{kPa})$ & $G^{\prime}(\mathrm{kPa})$ & $G^{\prime \prime}(\mathrm{Pa})$ \\
\hline $5 / 0.003$ & $1.52 \pm 0.24$ & $0.15 \pm 0.06$ & $11.77 \pm 0.33$ & $0.26 \pm 0.08$ & $0.08 \pm 0.02$ & $11.5 \pm 2.7$ \\
\hline $10 / 0.2$ & $36.14 \pm 2.28$ & $12.34 \pm 0.45$ & $378.66 \pm 436.64$ & $21.32 \pm 0.79$ & $7.28 \pm 0.27$ & $45.1 \pm 8.1$ \\
\hline $20 / 0.3$ & $110.99 \pm 2.66$ & $37.91 \pm 0.45$ & $423.04 \pm 692.99$ & $74.88 \pm 5.13$ & $25.57 \pm 1.75$ & $347.8 \pm 395.7$ \\
\hline
\end{tabular}

The shear storage modulus $\left(G^{\prime}\right)$ and the shear loss modulus $\left(G^{\prime \prime}\right)$ of PA gels with three different acrylamide $\left(A A\right.$, monomer) $/ N, N^{\prime}$-methylenbisacrylamide $(B I S$, crosslinker) concentrations $(\% w / v)$ was measured by rotational rheometry. The PA gels were measured either 20 min after initiation of polymerization directly in the rheometer $(n=2)$ or after swelling equilibration (in PBS at room temperature overnight, $n=4)$. The Young's modulus $(E)$, which is more commonly used to describe substrate elasticity, was computed from the dynamic modulus $(G)$. The values are reported as mean \pm standard error

\section{Results}

\section{Substrate characterization}

In preliminary polymerization experiments, three different AA/BIS compositions have been selected to span the entire range of stiffness ( 1 to $100 \mathrm{kPa}$ ) obtainable with PA (data not shown). Time course measurements (in situ polymerization, Fig. 1) of the selected compositions showed that 20 min after initiation, the shear storage modulus $G^{\prime}$ leveled off into a plateau, thereby indicating the completion of the polymerization. Degassing the AA/BIS solution prior to polymerization neither changed the polymerization pattern nor the final plateau levels (data not shown). Based on this finding, $>4 \mathrm{~h}$ of polymerization were considered sufficient for substrate preparation. To be more representative of in vitro cell culture conditions, PA was swollen (equilibrium) in PBS. This caused a reduction in stiffness by 33 to $85 \%$ as compared to the corresponding in situ polymerized PA (Table 2).

Based on the values obtained for the three distinct compositions of swollen PA (Table 2), the substrates employed in this work are referred to as $0.3 \mathrm{kPa}$ (soft), $21 \mathrm{kPa}$ (intermediately stiff), and $75 \mathrm{kPa}$ (stiff). TCPS coated with type I collagen (TCPS w/CI) was considered to be sensed by cells as infinitely stiff substrates.

HAC attachment, initial adhesion strength, and proliferation

EDD HAC were seeded in chondrogenic medium on substrates of different stiffness and their initial response characterized in terms of attachment and initial adhesion strength. EDD HAC attachment was comparable on the three different PA substrates, but 17 to $28 \%$ lower (Fig. 2A, Kruskal-Wallis, Conover, $p \leq 0.05$ ) than on TCPS $\mathrm{w} / \mathrm{CI}$. Counterintuitively, the time course centrifugation assay (Fig. 2B) showed that the initial cell adhesion strength on TCPS w/CI was lower than on the PA substrates as it took two to three times longer $(21.4 \pm 5.8 \mathrm{~min}$, KruskalWallis, paired, Conover, $p<0.01$ ) to reach $\mathrm{t}_{50}$ (time point at which $50 \%$ of the cells remained adherent) on TCPS w/CI. Similar as for the attachment however, the initial adhesion strength on the PA substrates did not significantly differ from each other (soft, $6.8 \pm 2.8 \mathrm{~min}$; intermediately stiff, 9.6 $\pm 4.2 \mathrm{~min}$; stiff, $9.6 \pm 4.9 \mathrm{~min}$ ).

On the stiff and intermediately stiff PA, the adherently cultured EDD HAC accomplished three to four times more doublings than on soft substrates and TCPS w/CI (Fig. 3; Kruskal-Wallis paired (Conover); ${ }^{*} p<0.05$ ). Still, after 1 week of chondrogenic culture, the proliferation did not exceed 1 total doubling on any of the different substrates.

\section{HAC morphology}

EDD HAC which have been stimulated to re-differentiate in chondrogenic medium for 7 days were found to be evenly distributed on all substrate surfaces at a cell density that limited the extent of cell-cell contacts. This cell density was chosen on purpose to favor the observation of cell-substrate effects. On soft PA displayed a distinct rounded morphology with limited spreading (Fig. 4C). In contrast, on intermediately to infinitely stiff substrates, HAC were fully spread and assumed an average spreading area, which was more than 3.2-fold higher than on the soft substrate (Fig. 4A, one-way ANOVA, Tukey HSD; $p<0.001)$. That on the soft substrates, HAC tended to assume a round morphology was also reflected by the shape factor $\Phi_{\mathrm{A}}$, which was 40 to $50 \%$ higher than on intermediately to infinitely stiff substrates (Fig. 4B, one-way ANOVA, Tukey HSD; $p<0.001)$. On intermediately stiff to infinitely stiff substrates, the average spreading area and shape factor of HAC was found to be similar.

\section{HAC actin cytoskeleton organization}

EDD HAC which have been stimulated to re-differentiate were fluorescently labeled for F-actin and vinculin to reveal parts of the cytoskeleton (Fig. 5), which are known to greatly determine cell morphology. On the soft substrate where the cells remained spherical, F-actin mainly localized 
A

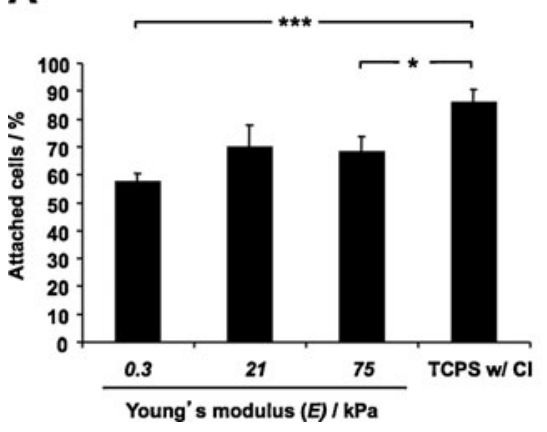

Fig. 2 A Attachment of EDD HAC on the corresponding substrate: 0.3 (soft), 21 (intermediately stiff), and $75 \mathrm{kPa}$ (stiff) indicate the substrate elasticity (Young's modulus) of the three contrasting, type I collagen functionalized polyacrylamide substrates. Tissue culturetreated plastic coated with type I collagen (TCPS w/CI) served as an infinitely stiff control. The asterisks indicate significant differences (Kruskal-Wallis paired (Conover); ${ }^{*} p<0.05$ and $* * * p<0.001 ; n=6$ ). B The initial adhesion strength of HAC on soft (blue), intermediately

cortically (Fig. 5A and B). On intermediately stiff to stiff substrates however, HAC were spread, flattened and displayed a fibrillar F-actin organization into stress fibers (Fig. 5C, D, E, F). On all substrates, vinculin was dispersed throughout the entire cytoplasm but appeared most intense in the perinuclear region. Aggregation of vinculin into small clusters was only detected in lamellipodial regions cultured on intermediately stiff to stiff substrates (Fig. 5D and F). On TCPS w/CI, F-actin assembly and vinculin clustering was identical to that on intermediately stiff to stiff substrates (data not shown).

Gene expression

After 7 days of chondrogenic culture, the initially seeded EDD HAC (Fig. 6A, dashed line) TCPS showed a

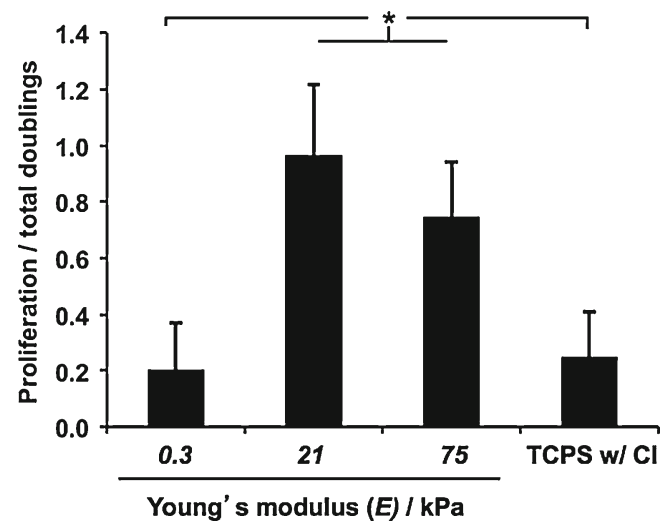

Fig. 3 Proliferation (total doublings) of EDD HAC after 7 days of chondrogenic culture on the corresponding substrate: $0.3 \mathrm{kPa}$ (soft), $21 \mathrm{kPa}$ (intermediately stiff), and $75 \mathrm{kPa}$ (stiff). Tissue culture-treated plastic coated with type I collagen (TCPS w/CI) served as an infinitely stiff control. The asterisk indicates significant differences (KruskalWallis paired (Conover); ${ }^{*} p<0.05 ; n=6$ )
B

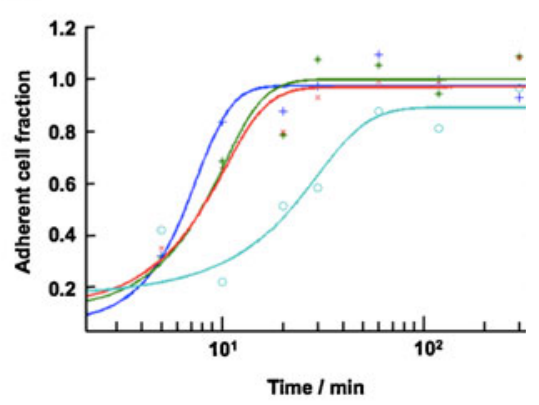

stiff (green), stiff (red) and TCPS w/CI as infinitely stiff (turquoise; open circles) substrate was assessed using a centrifugation assay. Based on a sigmoidal fit through the time course points of adherent cells, the time point at which $50 \%$ of the seeded cells remained adherent $\left(t_{50}\right)$ was calculated and served as a measure to approximate the initial adhesion strength on the corresponding substrate $(n=6$, two different donors. For interpretation of the references to color please see the web version of this article)

strong general up-regulation of type II collagen mRNA (Fig. 6A), which was inversely correlated with substrate stiffness. On the soft and the intermediately stiff substrates, type II collagen mRNA expression was 18.1and 6.2-fold higher than on infinitely stiff substrates (Kruskal-Wallis paired (Conover); $p<0.05$ ). Strikingly, on the soft substrates similar expression levels were reached as in aggregate culture, which were found to be highest. To investigate whether the soft substrate per se would be sufficient to up-regulate type II collagen mRNA, the soluble differentiation stimulus TGF- $\beta 1$ was omitted from the chondrogenic medium. In the absence of TGF- $\beta 1$ however, type II collagen mRNA expression remained on levels comparable to that of initially seeded EDD HAC (Fig. 6).

Although far less dramatic than for type II collagen, the initially seeded EDD HAC (Fig. 6B, dashed line) showed an increase in type I mRNA after 7 days of chondrogenic culture on soft (12.2-fold) and intermediately stiff substrates (6.2-fold; Kruskal-Wallis paired (Conover); $p<0.05$ ). TGF$\beta 1$ induced type I collagen mRNA expression did not appear to be modulated by matrix elasticity and remained at levels comparable to those of initially seeded EDD HAC. In absence of TGF- $\beta 1$ type I collagen mRNA on TCPS $\mathrm{w} / \mathrm{CI}$ remained on initial levels and but tended to be lower on the PA substrates and in aggregates (3.9 to 9.6-fold).

\section{Type II collagen protein expression}

In a last step we assessed, whether the re-differention of EDD HAC observed by type II collagen also translates into the corresponding protein expression. To increase the chance of matrix accumulation, chondrogenic culture was prolonged to 14 days, as it is standard for EDD HAC redifferentiation culture in pellets. Moreover, the initial cell 
Fig. 4 Average area $\mathrm{A}$ and shape factor $\Phi_{\mathrm{A}} \mathbf{B}$ of EDD HAC cultured in chondrogenic medium on the corresponding substrate: $0.3 \mathrm{kPa}$ (soft), $21 \mathrm{kPa}$ (intermediately stiff) and $75 \mathrm{kPa}$ (stiff). Tissue culture-treated plastic coated with type I collagen (TCPS w/CI) served as an infinitely stiff control. The shape factor was computed from the cell area and perimeter and can range from zero for starfish-shaped cells to 1 for perfectly round-shaped cells. The asterisks indicate significant differences (one-way ANOVA, Tukey HSD; $* * * p<$ $0.001 ; n$ from left to right $=55$, 67, 61, 79). C Representative phase contrast images of EDD HAC on corresponding substrates as described above. Scale bar $200 \mu \mathrm{m}$
A

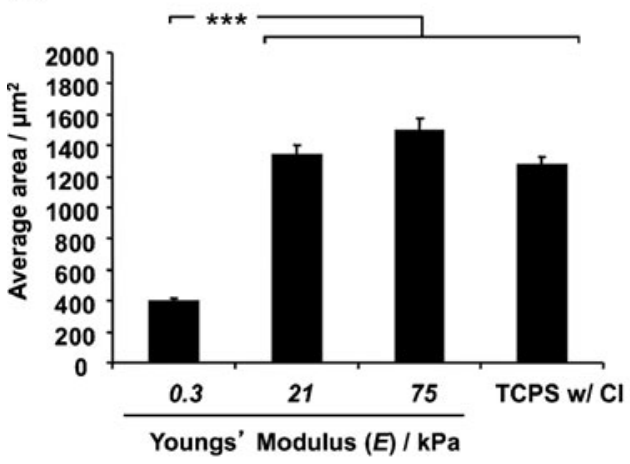

B
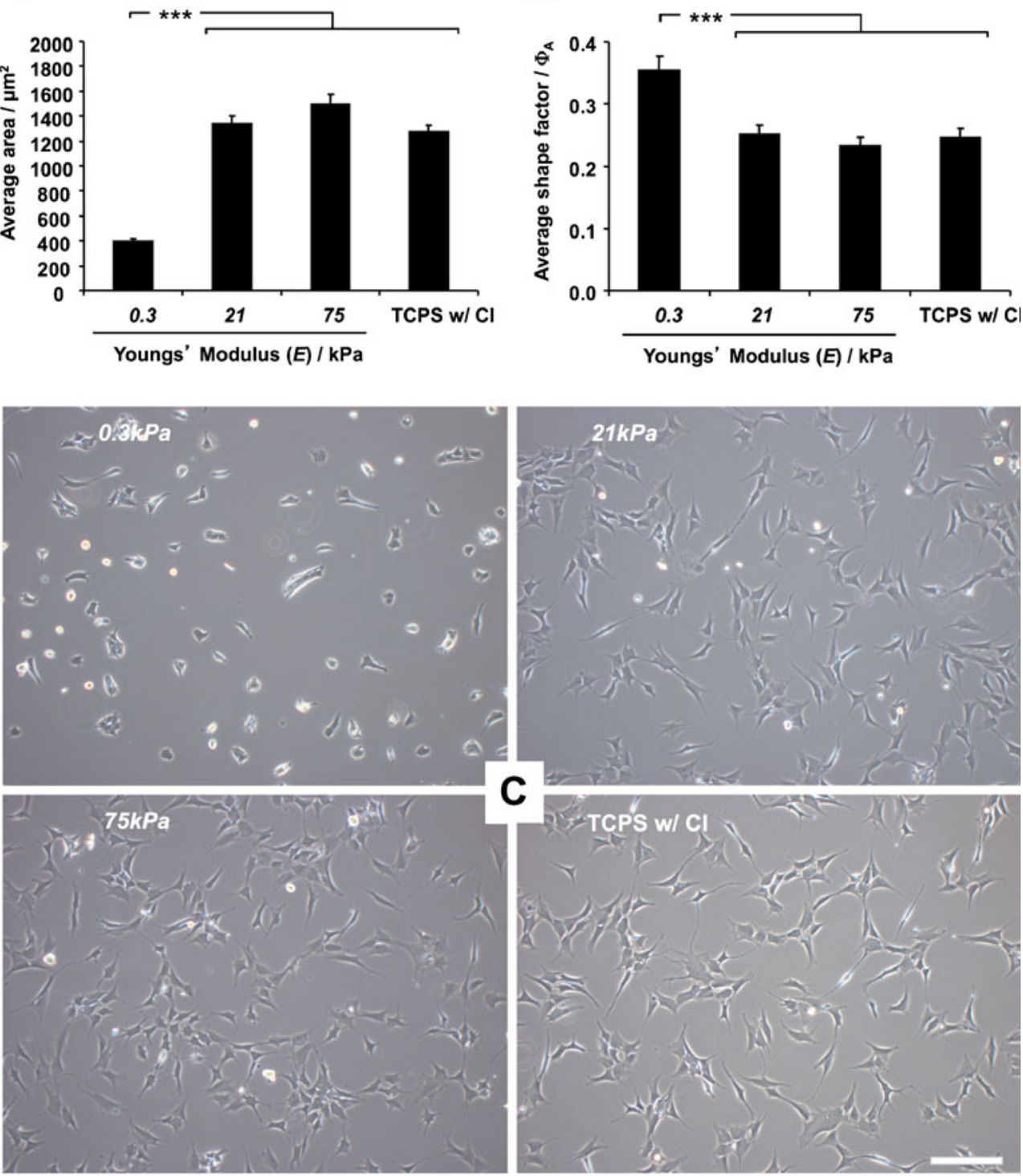

density was increased to allow for cell-cell contact, which is required for full chondrogenic differentiation. However, type II collagen protein expression remained undetectable by immunofluorescence and western blot analysis (data not shown).

\section{Discussion}

In this work we demonstrated that by reducing the stiffness of type I collagen-coated PA substrates it is possible to (1) support the maintenance of a spherical morphology of EDD $\mathrm{HAC}$, in which actin stress fibers and focal adhesions are absent and (2) to enhance HAC re-differentiation, independently of the number of doublings. Importantly, however, the latter effect was only observed in the presence of the chondrogenic stimulus TGF- $\beta 1$.
The elastic modulus describes the ability of a material to deform elastically (strain) when a force (stress) is applied to it, is quantified as a ratio of stress to strain and expressed in Pascals (Pa). Depending on the mode of measurement, the elastic modulus is commonly reported either as Young's modulus ( $E$, uniaxial stress over the uniaxial strain, e.g. indentation) or shear storage modulus $\left(G^{\prime}\right.$, deformation of shape at constant volume, e.g. shearing), given the Hooke's law holds for the material described. Since PA hydrogels have a vanishingly low viscous component (shear loss modulus $G^{\prime \prime}$ ) [34] their elasticity can adequately be described by either $E$ or $G^{\prime}$. As a fact, the elastic component of PA $\left(G^{\prime}\right)$ was always higher (6.9- to 161.4-fold) compared to its viscous component $G^{\prime \prime}$.

The shear storage modulus appears appropriate in the context of this work, since cells like i.e. fibroblasts are known to probe substrate mechanoproperties by shearing 

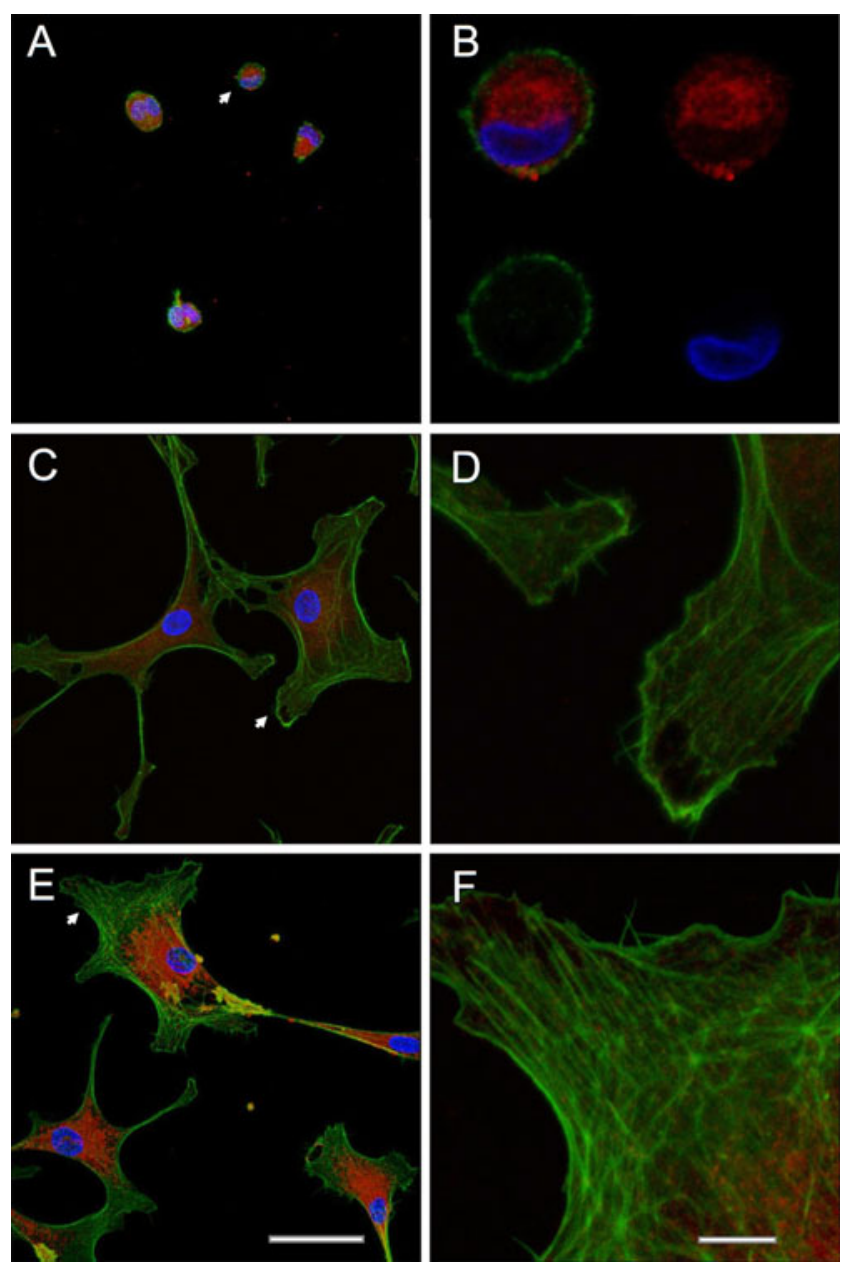

Fig. 5 Actin cytoskeleton organization of EDD HAC after 7 days of culture in chondrogenic medium on the corresponding substrate: $0.3 \mathrm{kPa}$ (A, B: soft), $21 \mathrm{kPa}(\mathbf{C}, \mathbf{D}$ : intermediately stiff) and $75 \mathrm{kPa}$ (E, F: stiff). Representative confocal laser-scanning microscopy images show false colors for F-actin (green), vinculin (red) and nuclei (blue). Images in the right hand column show details (indicated by the white arrow) of the corresponding image to their left. B Shows a merge and the separate channels of a mid section through a single HAC with spherical morphology (as indicated by the arrow in image A). Scale bars are $50 \mu \mathrm{m}$ for the left column and $10 \mu \mathrm{m}$ for the right column

[35]. However, the Youngs' modulus was preferred based on its wide spread use in the field of mechanobiology.

The density of surface-bound collagen has previously been shown to be independent of PA gel stiffness [36] and to have no influence on the elastic modulus of the substrate [7].

Type I collagen-coated tissue culture-treated plastic (TCPS w/CI) and glass served as substrates which are several orders of magnitudes stiffer (approximately $3 \mathrm{MPa}$ ) than the stiffest polyacrylamide substrate $[9,21,37]$.

HAC attachment on the different PA substrates was similar and is consistent with values previously found under expansion conditions [38]. The finding that HAC attachment on TCPS w/CI was higher than in all other PA substrates could be attributed to the different modalities of protein immobilization. In fact, while PA was functionalized with CI by chemisorption (sulfo-SANPAH crosslinker), TCPS was coated with $\mathrm{CI}$ by physisorption. Both approaches are known to influence native biological protein activity, and are likely do so in a distinct but unpredictable manner [39], which prompts for carefully comparing TCPS and PA in this regard. Indeed, we found that shortly after seeding, the adhesion strength of HAC on soft to stiff PA substrates was the similar; while on TCPS w/ $\mathrm{CI}$ it was significantly lower. This slightly faster adherence on the PA substrates could be due to a more flexible collagen presentation on the PA substrates, which could have allowed for a more immediate interaction with HAC integrins. However, since ultimately the attachment of HAC was in a comparable range on all substrates, we accepted TCPS w/CI for a direct comparison with PA.

HAC proliferation in defined, serum-free chondrogenic culture medium was very low on both soft and infinitely stiff substrates and allowed us to avoid proliferation, which was observed in a similar study hat was done in a basal medium containing $10 \%$ serum [11]. This enables us to exclude proliferation associated de-differentiation [12] which may have masked the analysis of matrix elasticity as a direct differentiation cue in the aforementioned study.

A significant effect of the soft substrates is, that they promoted a round cell morphology as it can be achieved by cytoskeleton disruption or cell embedding in a gel [40-43]. In fact, HAC cultured on soft PA substrates in chondrogenic medium displayed characteristic features of chondrocytes in situ [40-43], including a round/spherical morphology, cortical actin filament organization and the lack of focal adhesions. Moreover, the morphological observations in the present work were in good agreement with a complementary study, which assessed the maintenance of porcine and bovine chondrocytes in response to matrix elasticity $[11,20]$. Intermediately stiff to infinitely stiff substrates allowed for cell spreading, actin stress fiber formation, and focal adhesion assembly, which is accompanied by a reduced type II collagen mRNA expression. This suggests for the activation of Rho GTPases, which are well characterized upstream regulators of the actin cytoskeleton $[44,45]$ and have been found to influence chondrocyte differentiation and function. More specifically, RhoA and its effector ROCK suppress cortical actin organization, lead to stress fiber formation, the maturation of focal adhesions and inhibit chondrogenesis through the suppression of the expression of the main cartilage specific transcription factor Sox9 [46-48].

Matrix elasticity per se has been demonstrated to be sufficient for directing lineage specification of MSC [4], but it could not induce re-differentiation of HAC. This suggests that although HAC expanded in the presence of specific growth factors, assuming progenitor-like traits [4], 


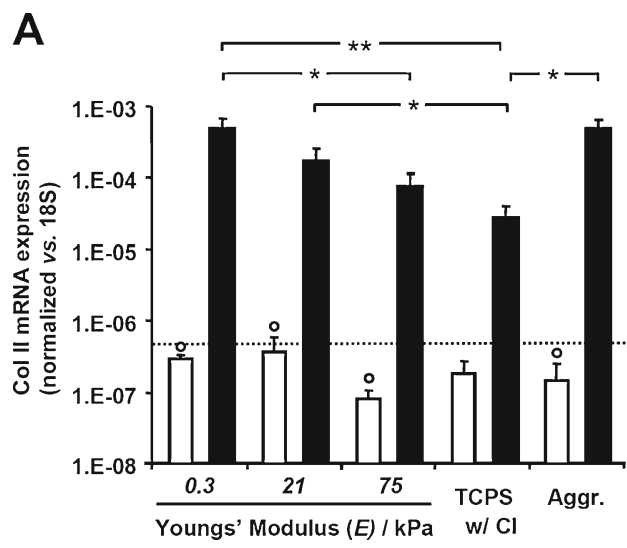

Fig. 6 Type II (A) and I (B) collagen mRNA expression levels of EDD HAC after 7 days of culture in chondrogenic medium without (white bars) or with (black bars) TGF- $\beta 1$ on the corresponding substrate: $0.3 \mathrm{kPa}$ (soft), $21 \mathrm{kPa}$ (intermediately stiff), and $75 \mathrm{kPa}$ (stiff). Tissue culture-treated plastic coated with type I collagen (TCPS w/CI) served as infinitely stiff control; and HAC aggregate cultures (Aggr.) as positive controls. All values are normalized vs. the housekeeping gene

they might not fully acquire the degree of plasticity of MSC. Although not additional, substrate elasticity was able to modulate the soluble chondrogenic stimulus provided by TGF- $\beta 1$. This suggests for a cross-talk between soluble and mechanical signals and implies that depending on the mechanical compliance of their environment, cells might respond differently to the same soluble signal. This has first been demonstrated for contractile fibroblasts, which only were able to maintain/generate TGF- $\beta 1$-induced $\alpha$ smooth muscle actin $(\alpha \mathrm{SMA})$, when cultured on stiff $(20 \mathrm{kPa})$ but not on soft $(8 \mathrm{kPa})$ collagen gels [5].

The strong up-regulation of type II collagen mRNA in HAC on soft substrates is in agreement with findings of a previous study using porcine chondrocytes [10]. However, the differences in type II collagen mRNA expression between the softest and the stiffest substrate were found to be more pronounced as compared to those reported by Schuh et al. [10]. This could be due to the fact that the softest substrate $(4 \mathrm{kPa})$ used by Schuh et al. [11] was more than tenfold stiffer than the soft substrates $(0.3 \mathrm{kPa})$ employed in the present study. Alternative possible explanations are the difference in cell type (human vs. porcine) and/or to the aforementioned proliferation associated de-differentiation.

That we were not able to detect extracellular type II collagen by fluorescence microscopy in HAC cultured on PA substrates (data not shown) could be due to 2D culture format, which likely does not support the retention of excreted matrix proteins. Additionally, intracellular type II collagen (lysates of harvested cells) also remained undetectable. Likely, its intracellular concentrations were too low for detection by western blot analysis. Thus, culture models in which excreted extracellular matrix proteins can be accumulated should be employed in future (see below).

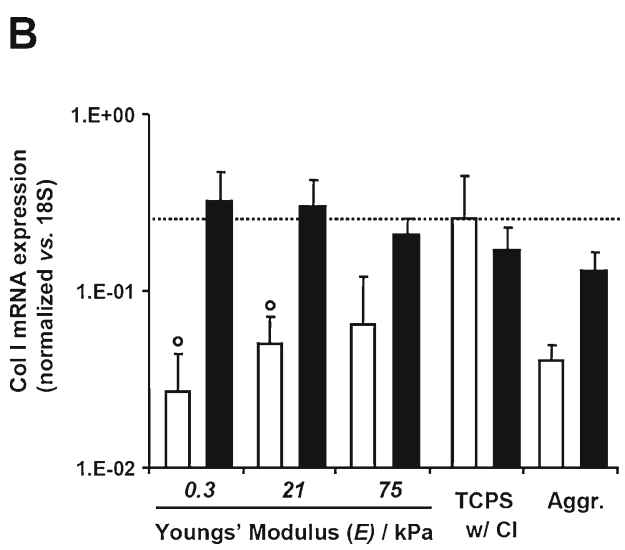

$18 \mathrm{~S}$ and the dashed line represents the mRNA expression level in EDD HAC prior to chondrogenic culture. Asterisks above the brackets indicate a significant difference between two corresponding bars (Kruskal-Wallis paired (Conover); ${ }^{*} p<0.05$ and ${ }^{*} p<0.01 ; n=15$; four donors). Open circles above the white bars indicate a significant difference vs. $(+)$ TGF- $\beta 1$ on the same substrate (Mann-Whitney, $U$ test, two-tailed; $n=4, p<0.05$ )

Strikingly, the stiffness of soft substrates matches very well with the $E$ modulus $(\sim 0.9 \mathrm{kPa})$ measured in single mesenchymal stem cells [49]. At the onset of chondrogenesis in the developing limb bud, mesenchymal stem cells aggregate into structures known as mesenchymal condensations [50]. Consequently, MSC can be expected to greatly determine the mechanical compliance of this developmental microenvironment. Thus, the soft substrates may have mimicked the mechanical aspect of this developmental microenvironment and could explain why the re-differentiation of EDD HAC was supported thereon.

In chondrogenic medium, type I collagen mRNA expression by HAC was rather high in both aggregates and on soft substrates. In the developing limb, collagen I plays an important role during mesenchymal condensation and is downregulated as chondrogenesis progresses [25]. This finding could reflect that 7 days of re-differentiation is still quite short and thus mirrors aspects of an early developmental stage.

The precise mechanisms by which substrate elasticity influences cell behavior were not subject of this study and in general are still elusive [51]. Still, the number of studies providing mechanistic insight into mechanotransduction is growing and has led to the proposal of different models.

An extracellular model describes that if cell generated tension meets resistance from the ECM, it allows to free/ activate latency-associated protein (LAP) bound growth factors (i.e., TGF- $\beta 1$ ) which are sequestered in their inactive form throughout the ECM [52]. However, it seems unlikely, that matrix elasticity modulated the effect of TGF- $\beta 1$ by this mechanism, since (1) the chondrogenic medium was supplemented with free/active TGF- $\beta 1$ and (2) type I collagen (immobilized on the PA substrates) is not known to possess a TGF- $\beta 1$ or a LAP interaction site. 
Other models focus on intracellular effects of matrix elasticity. The strain within a cell is manifested as protein extension, domain unfolding, and or protein-dissociation that relieves stress and/or creates sites for new binding partners, which elicits a chemical diffusion based signaling [51]. In more visionary models, external forces are thought to propagate into the cell nucleus through cell surface transmembrane receptors that couple the ECM to cytoskeletal networks, which in turn link to nuclear scaffolds, nucleoli, chromatin, and DNA. This channeling of forces across discrete load-bearing cytoskeletal filaments might promote changes in the shape, folding or kinetics of specific, loadbearing molecules or might modify higher-order chromatin organization and thereby alter nuclear protein-self-assembly, gene transcription, DNA replication or RNA processing [53]. This latter model would suggest that tissue elasticity as a mechanical cue influences genome organization and nuclear matrix composition, which in turn might prime cells to react differently to the same soluble signal.

Although the two dimensional (2D) PA gels employed in the present work offered a proof-of-principle of the regulatory role of substrate elasticity on chondrogenesis, future experiments should be performed in a three dimensional environment (3D). 3D culture systems possess fundamental advantages over 2D models as they allow cells to adopt their native morphology, increase cell-cell interactions and facilitate physiological contacts with the ECM $[54,55]$. A possible 3D model could be related to the one introduced by Schuh et al. using RGD-peptide modified agarose of different stiffness [56], whereby lower stiffness and the presence of TGF- $\beta 1$ should be introduced.

In this work, the adhesion of HAC was restricted to type I collagen, which could be substituted by other ECM proteins as, i.e. type II collagen, which has been shown to support the native morphology of chicken epiphyseal chondrocyte [57]. This suggestion also finds support by the fact that myogenic and osteogenic differentiation of human MSC can be directed by the interplay of substrate stiffness and type of adhesve ligand presented [58].

Alternatively to native proteins like type I collagen, engineered molecules (i.e., modified FN-fragments) or, as mentioned above, truncated proteins (i.e., RGDcontaining peptides) could also be used as cell-ligands and have previously been demonstrated to modulate the behavior of human fibroblasts and HAC, respectively $[38,59]$.

In conclusion, substrate elasticity modulated the TGF- $\beta 1$ stimulated re-differentiation of EDD HAC. Mechanical compliance is thus an important parameter to be coupled with the delivery of appropriate morphogens in designing biomaterials for cartilage engineering and repair.
Acknowledgments We would like to thank Peter Fischer and Varvara Mitropoulos from the ETHZ, Matthias Lütolf from the EPFL for their help with the rheological measurements, and Esther Kohler from the University Freiburg for her help with the western blot analysis.

\section{References}

1. Vinatier C, Mrugala D, Jorgensen C, Guicheux J, Noël D. Cartilage engineering: a crucial combination of cells, biomaterials and biofactors. Cell Trends Biotechnol. 2009;27(5):307-14.

2. Richter W. Mesenchymal stem cells and cartilage in situ regeneration. J Intern Med. 2009;266:390-405.

3. Saha K, Pollock JF, Schaffer DVS, Healy KE. Designing synthetic materials to control stem cell phenotype. Current opinion chem. 2007;11:381-7.

4. Engler A, Sen S, Sweeney H, Discher DE. Matrix elasticity directs stem cell lineage specification. Cell. 2006;126:677-89.

5. Goffin J, Pittet P, Csucs G, Lussi J. Focal adhesion size controls tension-dependent recruitment of $\alpha$-smooth muscle actin to stress fibers. J cell. 2006;172(2):259-68.

6. Pelham RJ, Wang Y. Cell locomotion and focal adhesions are regulated by substrate flexibility. National Acad Sci. 1997;94:13661-5.

7. Engler AJ, Griffin MA, Sen S, Bönnemann CG, Sweeney HL, Discher DE. Myotubes differentiate optimally on substrates with tissue-like stiffness: pathological implications for soft or stiff microenvironments. J Cell Biol. 2004;166(6):877-87.

8. Peyton SR, Putnam AJ. Extracellular matrix rigidity governs smooth muscle cell motility in a biphasic fashion. J Cell Physiol. 2005;204(1):198-209.

9. Gilbert P, et al. Substrate elasticity regulates skeletal muscle stem cell self-renewal in culture. Science. 2010;329(5995):1078-81.

10. Kong HJ, Polte TR, Alsberg E, Mooney DJ. FRET measurements of cell-traction forces and nano-scale clustering of adhesion ligands varied by substrate stiffness. Proc Natl Acad Sci U S A. 2005;102(12):4300-5.

11. Schuh E, et al. Effect of matrix elasticity on the maintenance of the chondrogenic phenotype. Tissue eng Part A. 2010;16(4):1281-90.

12. Darling EM, Athanasiou KA. Rapid phenotypic changes in passaged articular chondrocyte subpopulations. J Orthop Res. 2005;23 (2):425-32.

13. Stockdale F, Abbott J, Holtzer S, Holtzer H. The loss of phenotypic traits by differentiated cells. Dev Biol. 1963;7 (1):293-302.

14. Marlovits S, Hombauer M, Truppe M. Changes in the ratio of typeI and type-II collagen expression during monolayer culture of human chondrocytes. J Bone. 2004;86:286-95.

15. Aulthouse AL, et al. Expression of the human chondrocyte phenotype in vitro. Vitro Cell Dev Biol J Tissue Culture Assoc. 1989;25(7):659-68.

16. Barbero A, Ploegert S, Heberer M, Martin I. Plasticity of clonal populations of dedifferentiated adult human articular chondrocytes. Arthritis Rheum. 2003;48(5):1315-25.

17. Wolf $\mathrm{F}$, et al. Cartilage tissue engineering using pre-aggregated human articular chondrocytes. Cells Mater. 2008;16:92-9.

18. Barbero A, Grogan S, Schäfer D, Heberer M, Mainil-Varlet P, Martin I. Age related changes in human articular chondrocyte yield, proliferation and post-expansion chondrogenic capacity. Osteoarthr Cartil/OARS, Osteoarthr Res Soc. 2004;12(6):47684.

19. Jakob M, et al. Specific growth factors during the expansion and redifferentiation of adult human articular chondrocytes enhance chondrogenesis and cartilaginous tissue formation in vitro. J Cell Biochem. 2001;81(2):368-77. 
20. Genes NG, Rowley JA, Mooney DJ, Bonassar LJ. Effect of substrate mechanics on chondrocyte adhesion to modified alginate surfaces. Arch Biochem Biophys. 2004;422(2):161-7.

21. Solon J, Levental I, Sengupta K, Georges PC, Janme Pa. Fibroblast adaptation and stiffness matching to soft elastic substrates. Biophys J. 2007;93(12):4453-61.

22. Beningo KA, Lo C-M, Wang Y-L. Flexible polyacrylamide substrata for the analysis of mechanical interactions at cell-substratum adhesions. Methods Cell Biol. 2002;69:325-39.

23. Georges PC, Janmey Pa. Cell type-specific response to growth on soft materials. J Appl Physiol (Bethesda, MD: 1985). 2005;98 (4):1547-53.

24. Pierschbacher M, Ruoslahti E. Cell attachement activity of fibronectin can be duplicated by small synthetic fragments of the molecule. Nature. 1984;309(5963):30-3.

25. Dessau W, von der Mark H, von der Mark K, Fischer S. Changes in the patterns of collagens and fibronectin during limb-bud chondrogenesis. J Embryol Exp Morphol. 1980;57:51-60.

26. Beningo KA, Wang Y-L. Flexible substrata for the detection of cellular traction forces. Trends Cell Biol. 2002;12(2):79-84.

27. Takigawa T, Morino Y, Urayama K, Masuda T. Poisson's ratio of polyacrylamide (PAAm) gels. Polymer Gels Netw. 1996;4(1):1-5.

28. Channavajjala LS, Eidsath a, Saxinger WC. A simple method for measurement of cell-substrate attachment forces: application to HIV-1 Tat. J Cell Sci. 1997;110(2):249-56.

29. Maheshwari G, Wells a, Griffith LG, Lauffenburger Da. Biophysical integration of effects of epidermal growth factor and fibronectin on fibroblast migration. Biophys J. 1999;76(5):2814-23.

30. Reyes CD, García AJ. A centrifugation cell adhesion assay for high-throughput screening of biomaterial surfaces. J Biomed Mater Res Part A. 2003;67(1):328-33.

31. Abramoff $D$, Magalhaes $P$, Ram S. Image processing with ImageJ. Biophotonics Int. 2004;11(7):36-42.

32. Schuler M, et al. Fabrication of $\mathrm{TiO}_{2}$-coated epoxy replicas with identical dual-type surface topographies used in cell culture assays. J Biomed Mater Res Part A. 2009;88(1):12-22.

33. Stricker D. BrightStat.com: free statistics online. Comp Methods Program Biomed. 2008;92(1):135-43.

34. Calvet D, Wong JY, Giasson S. Rheological monitoring of polyacrylamide gelation: importance of cross-link density and temperature. Macromolecules. 2004;37(20):7762-71.

35. Harris AA, Wild P, Stopak D. Silicone rubber substrata: a new wrinkle in the study of cell locomotion. Science. 1980;208 (4440): 177-9.

36. Flanagan LA, Ju Y-E, Marg B, Osterfield M, Janmey PA. Neurite branching on deformable substrates. Dev Neurosci. 2002;13 (18):2411-5.

37. Engler AJ, Richert L, Wong JY, Picart C, Discher DE. Surface probe measurements of the elasticity of sectioned tissue, thin gels and polyelectrolyte films: correlations between substrate stiffness and cell adhesion. Surf Sci. 2004;570(1-2):142-54.

38. Vonwil D, et al. An RGD-restricted substrate interface is sufficient for the adhesion, growth and cartilage forming capacity of human chondrocytes. Eur Cells Mater. 2010;20:316-28.

39. Nakanishi K, Sakiyama T, Imamura K. On the adsorption of proteins on solid surfaces, a common but very complicated phenomenon. J Biosci Bioeng. 2001;91(3):233-44.

40. Yasui N, Osawa S, Ochi T, Nakashima H, Ono K. Primary culture of chondrocytes embedded in collagen gels. Exp Cell Biol. 1982;50(2):92-100.
41. Häuselmann HJ, Aydelotte MB, Schumacher BL, Kuettner KE, Gitelis SH, Thonar EJ. Synthesis and turnover of proteoglycans by human and bovine adult articular chondrocytes cultured in alginate beads. Matrix Stuttgart Germany. 1992;12(2):116-29.

42. Loty S, Forest N, Boulekbache H, Sautier JM. Cytochalasin D induces changes in cell shape and promotes in vitro chondrogenesis: a morphological study. Biol Cell Auspices Eur Cell Biol Organ. 1995;83(2-3):149-61.

43. Zanetti NC, Solursh M. Induction of chondrogenesis in limb mesenchymal cultures by disruption of the actin cytoskeleton. J Cell Biol. 1984;99(1 Pt 1):115-23.

44. Hall A, Nobes CD. Rho GTPases: molecular switches that control the organization and dynamics of the actin cytoskeleton. Philos Trans Royal Soc Lond Ser B, Biol Sci. 2000;355(1399):965-70.

45. Aspenström P. The Rho GTPases have multiple effects on the actin cytoskeleton. Exp Cell Res. 1999;25(1):20-5.

46. Akiyama H, Chaboissier MM-C, Martin JJF, Schedl A, De Crombrugghe $\mathrm{B}$. The transcription factor Sox 9 has essential roles in successive steps of the chondrocyte differentiation pathway and is required for expression of Sox 5 and Sox6. Genes Dev. 2002;16 (21):2813-28.

47. Bi W, Deng JM, Zhang Z, Behringer RR, de Crombrugghe B. Sox9 is required for cartilage formation. Nat Genet. 1999;22(1):85-9.

48. Lefebvre V, Huang W, Harley VR, Goodfellow PN, de Crombrugghe B. SOX9 is a potent activator of the chondrocyte-specific enhancer of the pro alpha1(II) collagen gene. Mol Cell Biol. 1997;17(4):233646.

49. Tan SCW, Pan WX, Ma G, Cai N, Leong KW, Liao K. Viscoelastic behaviour of human mesenchymal stem cells. BMC Cell Biol. 2008;9:40.

50. Hall BK, Miyake T. All for one and one for all: condensations and the initiation of skeletal development. BioEssays. 2000;22:138-47.

51. Zajac AL, Discher DE. Cell differentiation through tissue elasticity-coupled, myosin-driven remodeling. Curr Opin Cell Biol. 2008;20(6):609-15.

52. Wells RG, Discher DE. Matrix elasticity, cytoskeletal tension, and TGF- $\beta$ : the insoluble and soluble meet. Science. 2008;1:11-4.

53. Wang N, Tytell JD, Ingber DE. Mechanotransduction at a distance: mechanically coupling the extracellular matrix with the nucleus. Nat Rev Mol Cell Biol. 2009;10(1):75-82.

54. Abbott A. Biology's new dimension. Nature. 2003;424:870-2.

55. Cukierman E, Pankov R, Stevens DR, Yamada KM. Taking cellmatrix adhesions to the third dimension. Sci (New York, NY). 2001;294(5547):1708-12.

56. Schuh E, Hofmann S, Stok K, Notbohm H, Müller R, Rotter N. Chondrocyte redifferentiation in 3D: the effect of adhesion site density and substrate elasticity. J Biomed Mater Res Part A. 2012;100(1):38-47.

57. Shakibaei M, De Souza P, Merker HJ. Integrin expression and collagen type II implicated in maintenance of chondrocyte shape in monolayer culture: an immunomorphological study. Cell Biol Int. 1997;21(2):115-25.

58. Rowlands AS, George Pa, Cooper-White JJ. Directing osteogenic and myogenic differentiation of MSCs: interplay of stiffness and adhesive ligand presentation. Am J Physiol Cell Physiol. 2008;295 (4):C1037-44.

59. Grant RP, Spitzfaden C, Altroff H, Campbell ID, Helen J, Mardon HJ. Structural requirements for biological activity of the ninth and tenth FIII domains of human fibronectin. J Biol Chem. 1997;272 (10):6159-66. 